\title{
HISTÓRIA DA NOÇÃO DE MATRIZ: UMA RELEITURA SOB A LUZ DE NOVAS ABORDAGENS HISTORIOGRÁFICAS
}

\author{
Aline Bernardes \\ UNIRIO/UFRJ - Brasil \\ Tatiana Roque \\ UFRJ - Brasil \\ (aceito para publicação em maio de 2016)
}

\begin{abstract}
Resumo
Apresentamos a metodologia dos objetos e técnicas epistêmicas para a análise de fontes matemáticas. Esta metodologia foi adaptada da historiografia das ciências por Moritz Epple e possibilita explicitar e diferenciar os papéis que uma determinada noção matemática adquire em um ou mais episódios de pesquisa. Utilizaremos esta abordagem para investigar os diferentes papéis que a noção de matriz adquiriu em duas práticas elaboradas nos anos 1850 pelos matemáticos James Joseph Sylvester e Arthur Cayley, analisadas inicialmente por Frédéric Brechenmacher.
\end{abstract}

Palavras-chave: História das matrizes, objetos epistêmicos, técnicas epistêmicas.

\section{[HISTORY OF THE NOTION OF MATRICES: A REREADING BASED ON NEW HISTORIOGRAPHICAL APPROACHES]}

\begin{abstract}
We present the methodology of the epistemic object and techniques to analyze mathematical sources. This methodology was adapted from the historiography of science by Moritz Epple and enables to make explicit and differentiate the roles that a certain mathematical notion acquires in one or more episodes of research. We will use this approach to investigate the different roles that the notion of matrix acquired in two
\end{abstract}


practices developed in the 1850s by the mathematicians Cayley and James Joseph Sylvester e Arthur Cayley, already analyzed by Frédéric Brechenmacher.

Keywords: History of matrices, epistemic objetcs, epistemic thecniques.

\section{Introdução}

Ao comparar a ordem de exposição de alguns conceitos matemáticos com a ordem com a qual os mesmos surgiram na história, é comum se deparar com uma inversão. O conceito de matriz surgiu depois das noções de determinantes, sistemas lineares, transformações lineares e formas quadráticas.

O termo "matriz" foi introduzido pelo matemático britânico James Joseph Sylvester (1814-1897), em 1850, em um artigo publicado no Philosophical Magazine (SYLVESTER, 1850b), dedicado a um problema de natureza geométrica. Oito anos depois, Arthur Cayley (1821-1895) - contemporâneo e amigo de Sylvester - publicou uma memória em que definia as operações com matrizes e enunciava as propriedades dessas operações (CAYLEY, 1858).

As definições apresentadas por Sylvester e Cayley para o termo matriz são:

“(...) nós devemos começar, não com um quadrado, mas com um arranjo retangular de termos consistindo, suponha, de $m$ linhas e $n$ colunas. Isto não representará em si mesmo um determinante, mas, uma Matriz da qual podemos formar vários sistemas de determinantes por fixar um número $p$, e selecionar quaisquer $p$ linhas e $p$ colunas, os quadrados correspondendo ao que pode ser chamado de determinantes de p-ésima ordem”. (SYLVESTER, 1850b, p. 150, tradução nossa)

"O termo matriz pode ser usado em um sentido mais geral, mas nesta memória eu considero somente matrizes quadradas e retangulares, e o termo matriz sem qualificação deve ser interpretado como uma matriz quadrada; neste sentido restrito, um conjunto de quantidades arranjadas na forma de um quadrado, por exemplo,

$$
\begin{array}{ll}
\left(\begin{array}{lll}
a, & b, & c
\end{array}\right) \\
\left|\begin{array}{lll}
a^{\prime}, & b^{\prime}, & c^{\prime} \\
a^{\prime \prime}, & b^{\prime \prime}, & c^{\prime \prime}
\end{array}\right|
\end{array}
$$

é dito ser uma matriz. A noção de matriz surge naturalmente da notação abreviada para um conjunto de equações lineares, a saber, as equações 


$$
\begin{array}{lll}
X=a x & +b y & +c z \\
Y=a^{\prime} x & +b^{\prime} y & +c^{\prime} z \\
Z=a^{\prime \prime} x & +b^{\prime \prime} y & +c^{\prime \prime} z
\end{array}
$$

podem ser mais simplesmente representadas por

$$
\begin{aligned}
(X, Y, Z) & =\left(\begin{array}{lll}
a, & b, & c
\end{array}\right)(x, y, z) \quad \text { ". } \\
& \left|\begin{array}{lll}
a^{\prime}, & b^{\prime}, & c^{\prime} \\
a^{\prime \prime}, & b^{\prime \prime}, & c^{\prime \prime}
\end{array}\right|
\end{aligned}
$$

(CAYLEY, 1858, p. 17, tradução nossa).

Se apenas as definições forem analisadas, levando em conta o caráter universal da matemática e desconsiderando o contexto social, cultural e intelectual dentro do qual as definições acima foram colocadas, a tendência será ignorar os diferentes significados atribuídos à noção de matriz e se ater apenas às partes que apresentam traços comuns: "um arranjo retangular de termos" e "um conjunto de quantidades distribuídas na forma de um quadrado".

Um observador mais atento notará que Sylvester e Cayley associaram a noção de matriz a objetos matemáticos completamente distintos - determinantes e sistemas de equações lineares. Além disso, utilizaram esta noção em problemas matemáticos distintos. Cabe mencionar, ainda, que esses dois matemáticos ingleses compartilharam interesses comuns em alguns problemas ${ }^{1}$. O que os levou a conceber a noção de matriz associada a objetos distintos?

A perspectiva que desconsidera fatores como o contexto social, cultural e intelectual ao analisar uma fonte matemática, contribui para a visão, ainda muito comum, de que as noções matemáticas são entidades atemporais. Porém, ao investigar a produção do conhecimento matemático a partir de sua prática, é possível perceber que as noções matemáticas emergem em práticas específicas, situadas no tempo e no espaço, e se modificam dependendo das pesquisas com as quais se relacionam.

Neste trabalho, pretendemos analisar e comparar dois episódios da pesquisa sobre matrizes. Para tanto, apoiaremo-nos na interpretação histórica de Frédéric Brechenmacher ${ }^{2}$ (2006a), que discutiu as diferentes identidades e significações que a noção de matriz assumiu dentro das práticas elaboradas por James Joseph Sylvester, Arthur Cayley e Edward Weyr, entre 1850 e 1890.

No período acima, a Álgebra Linear ainda não existia como disciplina, o que só ocorreu nos anos 1930. Antes disso, os objetos que hoje constituem a Álgebra Linear eram vistos como pertencentes a domínios distintos, como a teoria das formas bilineares, a teoria

\footnotetext{
1 Sylvester e Cayley trabalharam em colaboração e lançaram as bases para a teoria dos invariantes (BRENCHENMACHER, 2006a).

2 Este historiador da matemática francês fez um profundo estudo sobre a história das matrizes, mais especificamente, sobre a história do Teorema de Jordan e da decomposição matricial em sua tese de doutorado (BRECHENMACHER, 2006b).
} 
dos determinantes e mesmo uma "teoria das matrizes". Brechenmacher discute o papel das práticas algébricas desenvolvidas em torno das matrizes que serviram para fundar um caráter unificador da representação matricial, o que contribuiu para a constituição da Álgebra Linear.

Tendo como foco os objetos estudados e as ferramentas matemáticas empregadas, propomos uma releitura dos episódios que envolvem: i) o trabalho inicial de Sylvester e sua introdução do termo "matriz" e ii) a elaboração de um cálculo simbólico com as matrizes por Cayley. Nosso objetivo é investigar os papeis que a noção de matriz adquiriu em cada episódio. Com este fim, pareceu-nos interessante usar os conceitos de objetos epistêmicos e técnicas epistêmicas, que apresentaremos na próxima seção. No restante do artigo, descreveremos os dois episódios e, em cada um, identificaremos os objetos e as técnicas epistêmicas, bem como a configuração epistêmica que está em jogo.

\section{Objetos epistêmicos e técnicas epistêmicas}

Os conceitos de objetos epistêmicos e técnicas epistêmicas foram adaptados da historiografia das ciências por Moritz Epple para descrever e analisar episódios de pesquisa a partir da prática da matemática. Eles oferecem uma perspectiva historiográfica que possibilita contextualizar no tempo e no espaço os objetos e ferramentas envolvidos nos episódios investigados. Estes conceitos foram introduzidos por Hans-Jörg Rheinberger em seus estudos sobre a prática experimental de biologistas moleculares. Epple apresenta a sua interpretação do quadro metodológico desenvolvido por Rheinberger (1997) e sua adaptação para a história da matemática em (EPPLE, 1999), conforme citado por (EPPLE, 2004).

Objetos epistêmicos são os objetos sob investigação, as construções intelectuais ou ainda os processos que são referidos como objetos pelos cientistas. Eles são elementos da pesquisa que induzem questões, uma vez que estão sob investigação, porque são parcialmente ou vagamente compreendidos. Já as técnicas epistêmicas são procedimentos, técnicas que permitem determinar algumas das características vagas acerca dos objetos de pesquisa, possibilitam formular questões precisas sobre os objetos e fornecem ferramentas para resp onder às questões do problema ou algumas delas. Em geral, são produtos finais de atividades anteriores (EPPLE, 2004).

Para citar um exemplo, vamos considerar o texto Ars Magna de Cardano, a partir da tradução de Witmer (2007). Nele, o que está sob investigação são as soluções de equações expressas por radicais, em especial, a solução de equações cúbicas e a apresentação de métodos para transformar equações. Assim, considerando o episódio da pesquisa sobre a resolução de equações por radicais delimitado por este texto, o objeto epistêmico é composto pelas equações algébricas. Já as técnicas epistêmicas são compostas pelas regras da álgebra que se aplicam à resolução de equações conhecidas até o momento, bem como pelas proposições de Euclides empregadas para demostrar as regras.

Há, ainda, um terceiro conceito, o de configuração epistêmica, que é a totalidade dos recursos intelectuais envolvidos em um episódio de pesquisa. Tais recursos compreendem a linguagem matemática, as técnicas utilizadas, os tópicos de pesquisa e 
problemas abertos sob consideração em um episódio, todo o horizonte de ideias seguidas pelos pesquisadores envolvidos (EPPLE, 2004, p. 148).

Retomando o exemplo de Cardano, a configuração epistêmica inclui, dentre outros elementos, a chamada arte cossista ${ }^{3}$ de resolver equações, praticada pelos algebristas dos séculos XIV, XV e XVI. A álgebra árabe, bem como as contribuições de Scipione del Ferro, Tartaglia e Ludovico Ferrari, citadas pelo próprio Cardano, e suas resoluções de alguns tipos de equação também compõem a configuração epistêmica desse episódio.

A leitura de fontes históricas pelos conceitos descritos acima se mostra adequada quando se deseja comparar episódios da pesquisa matemática relacionadas à origem de uma noção matemática e/ou ao desenvolvimento simultâneo de uma teoria. O seu ponto forte está na dinâmica das configurações epistêmicas, uma vez que os objetos e técnicas epistêmicas não são imutáveis e atemporais, eles são localizados no tempo e no espaço. Isto permite destacar as diferenças ao comparar episódios de pesquisa.

O que é considerado objeto em um determinado episódio pode, mais tarde, se tornar uma técnica estável de pesquisa. Por exemplo, os logaritmos foram o objeto epistêmico de John Napier e Henri Briggs no início do século XVII e se tornaram uma técnica epistêmica para os astrônomos, dentre eles Johannes Kepler que construiu suas próprias tabelas logarítmicas (FAUVEL \& GRAY, 1987).

Técnicas aplicadas em determinadas situações podem se tornar problemáticas e, mais tarde, se constituir em objetos de investigação. Os números complexos e os números negativos ilustram essa situação. Eles eram considerados nos passos intermediários da resolução de equações por Cardano e seus contemporâneos, apesar de não serem aceitos como soluções (ROQUE, 2012). No caso das equações cúbicas irredutíveis, números complexos eram somados e podiam resultar em uma solução verdadeira (positiva). Quando a natureza desses números começou a ser discutida, podemos dizer que eles assumiram o papel de objetos epistêmicos, como, por exemplo, com Argand e Gauss. Por fim, objetos e técnicas podem trocar de lugar de um episódio à outro, ou podem desaparecer.

Epple (2004) utilizou esta metodologia para analisar duas contribuições influentes na topologia, no início do século passado. Trata-se de dois episódios simultâneos da pesquisa matemática sobre a teoria dos nós, cujos principais matemáticos envolvidos foram James W. Alexander e Kurt Reidemeister.

Kjeldsen (2009) também aplicou esta metodologia para analisar dois episódios simultâneos da pesquisa matemática, no final do século XIX, associados à origem da teoria da convexidade. O primeiro foi delimitado pelas pesquisas de Karl Hermann Brunn (18621939) sobre corpos convexos e o segundo pelas pesquisas de Hermann Minkowski (18641909).

A respeito da aplicabilidade da metodologia, Kjeldsen destaca que os conceitos adaptados por Epple favorecem a distinção de como os elementos que geram o problema e aqueles que geram as respostas funcionam, interagem e mudam no curso do trabalho de um matemático ou de um grupo de matemáticos.

\footnotetext{
${ }^{3}$ A tradução para o alemão da palavra "coisa" (incógnita) deu origem ao termo "coss", e a prática de resolver equações ficou conhecida como arte "cossista". Ao longo do século XVI, difundiram-se diversos textos "cossistas". Neles, o autor introduzia operações aritméticas e definia a notação que ia usar para as quantidades desconhecidas e suas potências e indicava como realizar operações com essas quantidades. (ROQUE, 2012)
}

RBHM, Vol. 16, n 31, p. 1-19, 2016 
A interpretação dos episódios acima, segundo a metodologia em questão, contribuiu para revelar as diferenças nas configurações epistêmicas de cada um, o que permitiu compreender e explicar porque as práticas elaboradas por Minkowski, e não as de Brunn, conduziram ao desenvolvimento da teoria da convexidade (KJELDSEN, 2009).

Os conceitos de objetos e técnicas epistêmicos também têm sido utilizados em propostas de ensino com abordagens históricas (JANKVIST, 2009, KJELDSEN \& BLOMНØJ, 2012). O objetivo é usar fontes históricas e pedir para que os estudantes/professores identifiquem os objetos sob investigação e as técnicas empregadas nesses textos.

Nas próximas seções, descrevemos os dois episódios da história da noção de matriz já anunciados e os analisamos sob a luz dos conceitos acima introduzidos.

\section{Episódio I: Sylvester e o problema dos contatos}

Sylvester nasceu em Londres. Atuou como professor de filosofia e matemática em universidades na Inglaterra e nos Estados Unidos por dois períodos de sua vida acadêmica. Em 1883, ocupou a cadeira de posição de Savilian Professor de Geometria em Oxford.

Foi um matemático bastante ativo. Ao longo de sua vida esteve ligado a várias academias de ciências - nos Estados Unidos, Göttingen, Naples, Boston, St Petersburg, Berlim, para citar algumas. Foi o primeiro editor do American Mathematical Journal e um grande contribuidor deste periódico ${ }^{4}$.

As publicações do (também) autor de poemas e sonetos estão reunidas em quatro volumes no The collected mathematical papers of James Joseph Sylvester e abrangem assuntos sobre análise finita, álgebra, determinantes, teoria da eliminação, teoria das equações, teoria das partições, teoria das formas, teoria dos invariantes e covariantes, matrizes, números hamiltonianos, etc5.

Entre 1850 e 1851, Sylvester publicou uma série de memórias ${ }^{6}$ analisando os tipos de interseções e contatos entre duas cônicas e entre duas quádricas.

Delimitaremos o episódio da pesquisa de Sylvester para análise neste trabalho a duas das memórias acima ${ }^{7}$ (SYLVESTER, 1850b, 1851b) tendo como foco o problema da classificação dos tipos de contatos entre duas cônicas, o qual nos referiremos daqui em diante como o problema dos contatos.

A principal contribuição de Sylvester em sua abordagem, em relação aos trabalhos de outros matemáticos sobre o mesmo problema, foi o recurso ao cálculo de determinantes (BRECHENMACHER, 2006a).

A denominação de contato é empregada para designar os pontos de interseção em que duas cônicas se tangenciam. Existem quatro tipos de contatos que podem ser caracterizados pela multiplicidade $(2,3 \mathrm{ou} 4) \mathrm{do}(\mathrm{s})$ ponto(s) de interseção no(s) qual(is) as cônicas se tangenciam (Figuras 1 e 2.)

\footnotetext{
${ }^{4}$ As informações biográficas de Sylvester se baseiam em (CAYLEY, 1889) e (PARSHALL, 1998).

${ }^{5}$ Para saber mais sobre a vida de Sylvester, veja a biografia escrita por Cayley (1889).

${ }^{6}$ As memórias mencionadas são (SYLVESTER, 1850a, 1850b, 1851a, 1851b, 1851c).

${ }^{7}$ Para saber mais sobre as pesquisas de Sylvester relacionadas ao problema dos tipos de contatos entre duas cônicas ou duas quádricas, veja (BRECHENMACHER, 2006a, 2006b).
} 


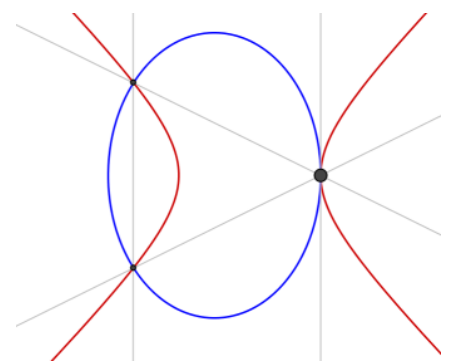

(a) Contato simples

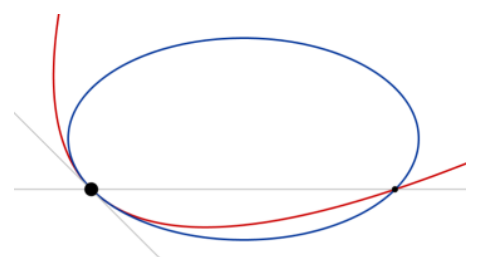

(b) Contato próximo

Figura 1: (a) Contato simples: um ponto de interseção duplo e (b) Contato próximo: um ponto de interseção triplo.

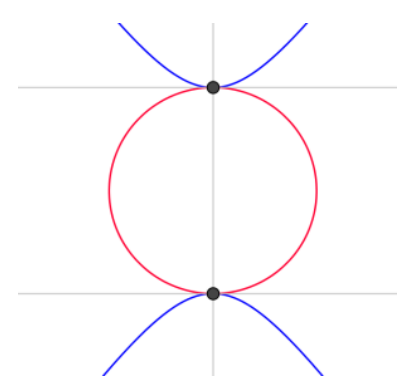

(a) Contato diplóide

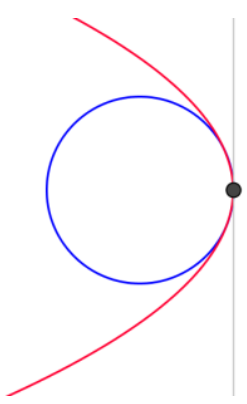

(b) Contato confluente

Figura 2: (a) Contato diploidal: dois pontos de interseção duplos e (b) Contato confluente: um ponto de interseção quádruplo.

As cônicas eram representadas por meio de equações homogêneas de segundo grau a três variáveis:

$$
U: a x^{2}+b y^{2}+c z^{2}+2 d x y+2 e x z+2 f y z=0
$$

com coeficientes reais. $\mathrm{O}$ polinômio homogêneo no lado esquerdo da equação era considerado a característica da cônica. Observamos que Sylvester estava lidando com cônicas projetivas.

No artigo (SYLVESTER, 1850a), publicado no The Cambridge and Dublin Mathematical Journal, Sylvester investigou a natureza dos pontos de interseção (reais/imaginários, finitos/infinitos) entre duas cônicas $U$ e $V$ e os tipos de pontos de contatos. Ele partia da equação cúbica em $\mu$ obtida ao igualar a zero o determinante de $U+$ $\mu V$. A equação $U+\mu V=0$ representa uma família de cônicas, todas passando pelos pontos de interseção de $U$ e de $V$. 
Quando as raízes $\mu$ de $|U+\mu V|=0$ são distintas, não há pontos de contato. Em caso de ocorrência de raízes duplas ou triplas (com multiplicidade algébrica 2 ou 3 , respectivamente), há pontos de contato.

Conhecendo a existência de quatro tipos de contatos, a análise da multiplicidade algébrica das raízes da equação cúbica de $|U+\mu V|=0$ se revelou um critério insuficiente para contemplar todos os casos. Para estabelecer as condições que distinguem dois tipos de contatos em cada caso em que a multiplicidade é diferente de 1 (quando há ocorrência de raiz dupla ou de raiz tripla) Sylvester comparou os fatores comuns no desenvolvimento polinomial dos coeficientes do seguinte determinante:

$$
\left|\begin{array}{cccc}
\frac{d^{2} W}{d \xi^{2}} & \frac{d^{2} W}{d \xi d \eta} & \frac{d^{2} W}{d \xi d \zeta} & p \\
\frac{d^{2} W}{d \eta d \xi} & \frac{d^{2} W}{d \eta^{2}} & \frac{d^{2} W}{d \eta d \zeta} & q \\
\frac{d^{2} W}{d \zeta d \xi} & \frac{d^{2} W}{d \zeta d \eta} & \frac{d^{2} W}{d \zeta^{2}} & r \\
p & q & r & 0
\end{array}\right|=A p^{2}+B q^{2}+C r^{2}+2 F q r+2 G r p+2 H p q
$$

“onde todos os coeficientes são funções quadráticas de $\mu$ [se referindo a $W=U+\mu V]$ e faça $A=0, B=0, C=0, F=0, G=0, H=0$;

cada uma desses seis equações em $\mu$ terá uma mesma raiz em comum”. (SYLVESTER, 1850a, p. 129, tradução nossa)

Na citação acima, Sylvester estava discutindo as condições para o caso de contato diplóide (Figura 2 (a)). Dentro deste quadro da resolução de um problema geométrico, em que condições eram estabelecidas para classificar os tipos de contatos entre duas cônicas, Sylvester introduziu as noções de determinantes menores, em outro artigo publicado no mesmo ano, no Philosophical Magazine:

"Imagine qualquer determinante colocado sob a forma de um arranjo ordenado quadrado de termos. Este quadrado pode ser considerado como divisível em linhas e colunas. Agora suponha que qualquer linha e qualquer coluna possa ser eliminada, nós obtemos desta forma um quadrado, um termo a menos em largura e em profundidade que o quadrado original; e por variar em qualquer maneira possível a seleção da linha e da coluna excluídos, nós obtemos, suponha que o quadrado original possua $n$ linhas e $n$ colunas, $n^{2}$ quadrados menores, cada um dos quais representará o que eu denomino um Primeiro Determinante Menor relativo ao determinante principal ou completo". (SYLVESTER, 1850b, p. 147, tradução nossa, grifo nosso)

No mesmo artigo, propriedades sobre os determinantes menores foram enunciadas e, em seguida, aplicadas para classificar o tipo de contato entre duas cônicas. A prática 
desenvolvida por Sylvester consistia em comparar os fatores comuns no desenvolvimento polinomial do determinante completo $|U+\mu V|$ e nos "primeiros determinantes menores":

“ Sejam agora U e V características de duas cônicas, isto é, cada uma, uma função de somente três letras, pode ser mostrado [...] que as diferentes espécies de contatos entre estas duas cônicas corresponderão a propriedades peculiares da característica composta $U+\mu V$.

Se o determinante desta função tem duas raizes iguais, as cônicas simplesmente se tocam; se ele tem três raízes iguais, as cônicas têm um contato simples de mais alta ordem, isto é, de mesma curvatura; se seus seis primeiros determinantes menores se anulam simultaneamente para o mesmo valor de $\mu$, as cônicas têm um contato duplo. Se o mesmo valor de $\mu$, o qual torna todos estes primeiros menores iguais a zero, for ao mesmo tempo não apenas uma raiz dupla mas uma raiz tripla de

$$
\square(U+\mu V)=0 \text {, }
$$

então as cônicas têm um contato simples da mais alta ordem possível, quase uma coincidência absoluta, isto é, eles se encontram em quatro pontos consecutivos." (SYLVESTER, 1850b, p. 148, tradução nossa.)

Sylvester estendeu o método acima para investigar as interseções entre duas quádricas (representadas por equações homogêneas de grau 2, a quatro variáveis) e, de forma mais geral, entre duas formas quadráticas ( $n$ variáveis). A generalização da técnica de extração de sistemas de determinantes menores foi baseada em uma representação em forma de tabela retangular à qual Sylvester denominou matriz (BRECHENMACHER, 2006a).

Ao final da última memória citada acima, Sylvester introduziu o termo matriz ${ }^{8}$. A noção de matriz surge, então, como uma tabela retangular, geradora de vários sistemas de determinantes menores, no seio de uma prática que consistia na extração destes menores que, em seguida, seriam utilizados para classificar o tipo de contato entre duas cônicas.

Observamos que, no artigo em que a noção de matriz foi introduzida, não foi apresentada uma representação em forma de tabela. Em outro artigo, publicado em 1851 no Philosophical Magazine, Sylvester fala novamente sobre o problema dos contatos, sem empregar matrizes diretamente. Em uma nota de rodapé, ele fala de matrizes em conexão com o determinante de uma função binária:

"[...] a teoria das funções quadráticas se mistura a uma teoria mais ampla de funções binárias, consistindo da soma de múltiplos de produtos binários formados por combinar cada um do conjunto de quantidades $x, y$ $e z$... com cada um do mesmo número de quantidades do conjunto $x^{\prime}, y^{\prime} e$ $z^{\prime} \ldots$ Por exemplo,

\footnotetext{
${ }^{8}$ Veja o extrato citado na introdução.
}

RBHM, Vol. 16, n 31 , p. 1-19, 2016 


$$
\begin{aligned}
& a x x^{\prime}+b x y^{\prime}+c x z^{\prime} \\
+ & a^{\prime} y x^{\prime}+b^{\prime} y y^{\prime}+c^{\prime} y z^{\prime} \\
+ & a^{\prime \prime} z x^{\prime}+b^{\prime \prime} z y^{\prime}+c^{\prime \prime} z z^{\prime}
\end{aligned}
$$

seria uma função binária e seu determinante (não mais, como em uma função quadrática, simétrico sobre dua diagonal) corresponderia à matriz quadrada

$$
\begin{array}{ccc}
a & b & c \\
a^{\prime} & b^{\prime} & c^{\prime} \\
a^{\prime \prime} & b^{\prime \prime} & c^{\prime \prime}
\end{array}
$$

(SYLVESTER, 1851b, p. 222, tradução nossa.)

Os tipos de contatos entre duas cônicas foram o principal elemento gerador de questões e, portanto, o objeto epistêmico deste episódio. Apesar de não se tratar de um novo objeto, uma vez que a classificação em quatro tipos de contatos já era conhecida e o problema de identificá-los já havia sido abordado por outros matemáticos como Plücker, em 1828 (BRECHENMACHER, 2006a, p. 10) - por métodos analíticos, Sylvester elaborou uma prática que permite identificar os tipos de contatos entre duas cônicas de uma nova maneira, com recurso ao cálculo de determinantes. Este é um exemplo de episódio de pesquisa onde o objeto sob investigação era conhecido e compreendido em alguma extensão.

Dentre os elementos que contribuíram para gerar respostas ao problema dos contatos, temos os determinantes, a ferramenta chave que permitiu estabelecer os critérios para a classificação. Assim, neste episódio, os determinantes estão no papel de técnicas epistêmicas. Além disso, a representação das cônicas por equações em coordenadas homogêneas, também contribuiu para gerar respostas, assim, também fazem parte das técnicas epistêmicas neste episódio da pesquisa de Sylvester.

Os determinantes menores foram introduzidos e propriedades sobre eles foram enunciadas, assim, eles também funcionaram como elementos geradores de questões:

“[...] nós devemos ser capazes de determinar o significado e o efeito de fatores comuns, um ou mais entrando nos sistemas sucessivos de determinantes menores, derivados do determinante completo de $U+\mu V$ ". (SYLVESTER, 1850b, p. 150, tradução nossa)

Ao mesmo tempo, eles foram os objetos fundamentais da técnica desenvolvida para classificar os tipos de contatos entre duas cônicas. Desta forma, eles desempenharam o papel de objeto epistêmico e ao mesmo tempo de técnica epistêmica.

As matrizes não foram o objeto de investigação de Sylvester nos trabalhos considerados por nós para delimitar este episódio de pesquisa. Elas foram introduzidas em conexão com a técnica de extração de menores que deixou de se apoiar no determinante completo, para se basear na tabela por trás do determinante, isto é, as matrizes ofereceram uma representação, a partir da qual os menores poderiam ser gerados. Assim, as matrizes 
contribuíram, em certa medida, para gerar a resposta do problema, isto é, para a classificação dos tipos de contatos entre duas cônicas. Logo, a noção de matriz está entre as técnicas epistêmicas do episódio em questão.

A configuração epistêmica de Sylvester compreende os objetos epistêmicos, as técnicas epistêmicas, bem como todo o quadro técnico da geometria analítica, da geometria projetiva e também o das formas quadráticas.

\section{Episódio II: Cayley e o cálculo simbólico com matrizes}

Cayley também foi um matemático inglês, nasceu em Richmond, Londres. Apesar de mostrar habilidade para matemática desde cedo, por razões financeiras, dividiu as suas atividades profissionais entre a lei e a matemática durante cerca de 15 anos, até que foi eleito para a posição de Sadlerian Professor da Universidade de Cambridge em 1863, cadeira que ele manteve pelo resto de sua vida.

Suas publicações, reunidas em 13 volumes nos The collected mathematical papers of Arthur Cayley, versavam sobre temas em geometria analítica, transformações lineares, matrizes, determinantes, teoria dos invariantes e covariantes, teoria das equações, cálculo, funções homogêneas, equações diferenciais, teoria dos grupos, etc ${ }^{9}$.

A generalização da prática de extração de sistemas de determinantes menores para determinantes de qualquer ordem colocou o problema de enumeração desses sistemas, o que chamou a atenção de Cayley para a noção de matriz e o levou a publicar três artigos sucessivos em 1855 (BRECHENMACHER, 2006a).

A noção de matriz foi utilizada por Cayley, pela primeira vez, no artigo Remarques sur la notation des functions algébriques (CAYLEY, 1855). Ele introduziu uma notação para matrizes, como sendo prática para representar sistemas lineares e formas quadráticas.

Em 1858, Cayley publicou uma memória sobre matrizes: A Memoir on the Theory of Matrices no Philosophical Transactions ${ }^{10}$ (CAYLEY, 1858). Nela, Cayley descreve o que é uma matriz, define as operações com matrizes, enuncia as propriedades das operações, enuncia e demonstra um resultado que ele se refere como "teorema notável" e apresenta aplicações desse resultado. O episódio da pesquisa de Cayley que será descrito e analisado neste trabalho será delimitado por esta memória.

Cayley definiu matriz ${ }^{11}$ como "um conjunto de quantidades organizadas em forma de quadrado" associando-a a uma notação abreviada de um conjunto de equações lineares. Tal notação é descrita como um conjunto de "funções lineares":

\section{"2. A notação}

\footnotetext{
${ }^{9}$ Para saber mais sobre a vida de Cayley, veja a biografia escrita por Forsyth (1895), o editor da coletânea de artigos de Cayley.

${ }^{10}$ Philosophical Transactions of the Royal Society of London.

${ }^{11}$ Veja o extrato citado na introdução.
}

RBHM, Vol. 16, n 31, p. 1-19, 2016 


$$
\begin{aligned}
& \left(\begin{array}{lll}
a, & b, & c
\end{array}\right)(x, y, z), \\
& \left|\begin{array}{lll}
a^{\prime}, & b^{\prime}, & c^{\prime} \\
a^{\prime \prime}, & b^{\prime \prime}, & c^{\prime \prime}
\end{array}\right|
\end{aligned}
$$

representa o conjunto de funções lineares:

$$
\left((a, b, c)(x, y, z),\left(a^{\prime}, b^{\prime}, c^{\prime}\right)(x, y, z),\left(a^{\prime \prime}, b^{\prime \prime}, c^{\prime \prime}\right)(x, y, z)\right) ” .
$$

(CAYLEY, 1858, p. 18, tradução nossa).

As operações com matrizes são definidas com base na relação entre matrizes e sistemas de equações lineares. A multiplicação de matrizes, por exemplo, é definida a partir da "composição de sistemas lineares", ou melhor, da composição de transformações lineares:

$$
\begin{aligned}
& \text { "11. As equações } \\
& (X, Y, Z)=(a, \quad b, \quad c)(x, y, z), \quad(x, y, z)=(\alpha, \quad \beta, \quad \gamma)(\xi, \eta, \zeta) \\
& \left|\begin{array}{ccc}
a^{\prime}, & b^{\prime}, & c^{\prime} \\
a^{\prime \prime}, & b^{\prime \prime}, & c^{\prime \prime}
\end{array}\right| \quad\left|\begin{array}{lll}
\alpha^{\prime}, & \beta^{\prime}, & \gamma^{\prime} \\
\alpha^{\prime \prime}, & \beta^{\prime \prime}, & \gamma^{\prime \prime}
\end{array}\right| \\
& \text { dão } \\
& (X, Y, Z)=(A, \quad B, \quad C)(\xi, \eta, \zeta)=(a, \quad b, \quad c)(\alpha, \quad \beta, \quad \gamma)(\xi, \eta, \zeta),, \\
& \left|\begin{array}{ccc}
A^{\prime}, & B^{\prime}, & C^{\prime} \\
A^{\prime \prime}, & B^{\prime \prime}, & C^{\prime \prime}
\end{array}\right| \quad\left|\begin{array}{ccc}
a^{\prime}, & b^{\prime}, & c^{\prime} \\
a^{\prime \prime}, & b^{\prime \prime}, & c^{\prime \prime}
\end{array}\right| \begin{array}{ccc}
\alpha^{\prime}, & \beta^{\prime}, & \gamma^{\prime} \\
\alpha^{\prime \prime}, & \beta^{\prime \prime}, & \gamma^{\prime \prime}
\end{array} \mid
\end{aligned}
$$

(CAYLEY, 1858, p. 20, tradução nossa.)

Em seguida, Cayley apresenta o resultado da composição de matrizes, ou multiplicação de matrizes, como:

$$
\begin{aligned}
& \left((a, b, c)\left(\alpha, \alpha^{\prime}, \alpha^{\prime \prime}\right) \quad(a, b, c)\left(\beta, \quad \beta^{\prime}, \quad \beta^{\prime \prime}\right) \quad(a, b, c)\left(\gamma, \gamma^{\prime}, \gamma^{\prime \prime}\right)\right)
\end{aligned}
$$

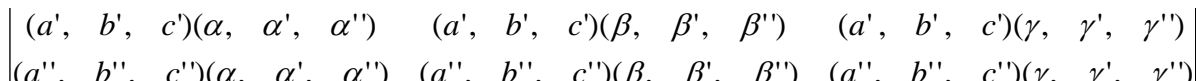

Eis aí a origem da multiplicação de matrizes, cuja definição moderna nem de longe permite vislumbrar.

A matriz nula e a matriz unidade (identidade) são definidas antes das operações. Após definir as operações com matrizes, Cayley enuncia as propriedades. Ele menciona a não validade da comutatividade para a multiplicação ou composição de matrizes e a validade da associatividade, dentre outras propriedades, e apresenta a noção de matriz inversa ou recíproca.

As contribuições de Cayley nesta memória não param na introdução de um cálculo simbólico com matrizes. Na primeira página da memória, Cayley anuncia um "teorema notável""12.

${ }^{12} \mathrm{O}$ "teorema notável" hoje é conhecido como Teorema de Cayley-Hamilton. 
"Eu obtenho um teorema notável de que qualquer matriz satisfaz uma equação algébrica de sua própria ordem, o coeficiente da mais alta potência sendo a unidade e, os das outras potências, funções dos termos da matriz, o último coeficiente sendo o próprio determinante".

(CAYLEY, 1858, p. 17, tradução nossa)

E grande parte da memória é dedicada a aplicações deste teorema, por exemplo: determinar uma matriz periódica e de ordem dada, conhecendo-se a periodicidade ${ }^{13}$ (isto é, determinar uma matriz $M$ de ordem $n$ satisfazendo $M^{p}=I$ (para algum valor de $p$ dado) e determinar todas as matrizes que comutam com uma matriz dada (isto é, dada a matriz $M$, determinar as matrizes $L$ que satisfazem $M L=L M$ ).

De acordo com Brechenmacher (2006a), a historiografia considera esta memória como um dos primeiros trabalhos sobre a teoria das álgebras associativas, enfatizando as leis das operações sobre as matrizes. No entanto, a motivação inicial de Cayley teria sido um problema já abordado por Charles Babbage: determinar a expressão das funções homográficas de periodicidade dada, ou seja, encontrar

$$
\varphi(x)=\frac{a x+\alpha}{b x+\beta} \text { tal que } \varphi^{n}(x)=x .
$$

Cayley fez uma observação no final da memória mencionando Babbage como já tendo investigado o problema da expressão das funções homográficas de periodicidade dada e relacionou algumas aplicações apresentadas na memória com esse problema. Babbage fez parte de uma geração de matemáticos da primeira metade do século XIX cuja corrente de pensamento foi designada como escola algébrica inglesa ${ }^{14}$. A abordagem desta escola tinha como ponto forte um simbolismo algébrico. Segundo Brechenmacher (2006a, p. 19), os problemas do cálculo de potências e raízes de matrizes retomam uma preocupação tradicional desta escola, após os trabalhos de Herschell, em 1813, sobre a notação dos operadores diferenciais e a propriedade $f^{n}(f(x))=f^{n+1}(x)$.

Para resolver os problemas acima, Cayley elaborou uma prática baseando-se em uma dupla interpretação da noção de matriz ora como um sistema de números, ora como uma "quantidade simples" (single quantity). Logo após definir a operação de multiplicar uma matriz por um número, Cayley introduziu a noção de "matriz quantidade simples".

\section{"10. Nós temos, em particular,}

\footnotetext{
${ }^{13} \mathrm{O}$ problema de determinar a expressão das funções homográficas de periodicidade dada pode ser resolvido com linguagem matricial. Para isso, basta considerar a matriz $M$ cujos elementos são os coeficientes de $\varphi$. Ao compor $\varphi$ $n$ vezes, a expressão obtida para os coeficientes coincide com os elementos de $M^{n}$.

${ }^{14}$ Os principais matemáticos associados a esta corrente são G. Peacock, D. F. Gregory, A. de Morgan, W. R. Hamilton e G. Boole (BRECHENMACHER, 2006a: 19).
} 


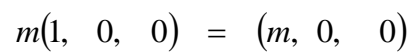

$$
\begin{aligned}
& \left|\begin{array}{lll}
1, & 0, & 0
\end{array}\right| \quad\left|\begin{array}{lll}
0, & m, & 0
\end{array}\right| \text {, } \\
& \begin{array}{lll}
0, & 0, & 1
\end{array}|\quad| \begin{array}{lll}
0, & 0, & m
\end{array} \mid
\end{aligned}
$$

ou, trocando a matriz no lado esquerdo pela unidade, nós podemos escrever

$$
\begin{array}{r}
m=\left(\begin{array}{lll}
m, & 0, & 0
\end{array}\right) \\
\left|\begin{array}{ccc}
0, & m, & 0 \\
0, & 0, & m
\end{array}\right|
\end{array}
$$

A matriz no lado direito é dita ser a quantidade simples $\boldsymbol{m}$ considerada como envolvendo a matriz unidade".

(CAYLEY, 1858, p. 20, tradução nossa, grifo nosso.)

Na demonstração do "teorema notável", Cayley interpreta a matriz dada $M$ ora como uma quantidade múltipla ora como uma quantidade simples, ou seja, ora como uma matriz, ora como um número:

"21. O teorema geral antes mencionado será melhor compreendido pelo desenvolvimento completo de um caso particular. Imagine uma matriz

$$
\begin{array}{r}
M=\left(\begin{array}{rl}
a, & b
\end{array}\right), \\
|c, \quad d|
\end{array}
$$

e forme o determinante

$$
\left|\begin{array}{cc}
a-M, & b \\
c, & d-M
\end{array}\right|,
$$

a expressão desenvolvida deste determinante é

$M^{2}-(a+d) M^{1}+(a d-b c) M^{0}$

[...] substituindo estes valores o determinante se torna igual a matriz zero $[\ldots]$ ”.

(CAYLEY, 1858, p. 23, tradução nossa)

Sobre o determinante formado, Cayley considera:

$$
\begin{aligned}
& \left(\begin{array}{ll}
a-M, & b
\end{array}\right)=\left(\begin{array}{ll}
a, & b
\end{array}\right)-M(1, \quad 0), \\
& |c, \quad d-M| \quad|c, \quad d| \quad|0, \quad 1|
\end{aligned}
$$

ou seja, "a matriz original, diminuída pela mesma matriz considerada como uma quantidade simples (escalar) envolvendo a matriz unidade (identidade)" (CAYLEY, 1858, p. 24).

Após a demonstração do "teorema notável", são apresentadas as aplicações, sendo a primeira delas o problema de determinar as raízes quadradas de uma matriz quadrada de 
ordem 2. Com base no mesmo resultado, Cayley desenvolveu uma prática de fatoração de polinômios de matrizes:

$$
\begin{aligned}
& \text { “[... } \quad M^{2}-(a+d) M+a d-b c=0 \\
& \text { e sejam } X_{i}, X_{i i} \text { as quantidades simples, raízes da equação } \\
& \left|\begin{array}{cc}
a-X, & b \\
c, & d-X
\end{array}\right|=0, \\
& \text { ou } \\
& X^{2}-(a+d) X+a d-b c=0 . \\
& \text { A equação satisfeita pela matriz pode ser escrita } \\
& \left(M-X_{i}\right)\left(M-X_{i i}\right)=0,
\end{aligned}
$$

[...] à primeira vista, deveríamos ter um dos fatores simples igual a zero, o que obviamente não é o caso, para uma tal equação significaria que a matriz perfeitamente indeterminada $M$ é igual a uma quantidade simples, considerada como envolvendo a matriz unidade. A explicação é que cada um dos fatores simples é uma matriz indeterminada, de fato $\left(M-X_{i}\right)$ representa a matriz

$$
\left|\begin{array}{cc}
a-X_{i}, & b \\
c, & d-X_{i}
\end{array}\right|,
$$

e o determinante desta matriz é igual a zero. O produto de dois fatores é, assim, igual a zero sem que nenhum dos seus fatores seja igual a zero."

(CAYLEY, 1858: 26-27)

No extrato acima, Cayley descreve a fatoração de polinômios em matrizes e discute o fato de que um produto de matrizes igual à matriz nula não implica que qualquer dos fatores do produto seja igual à matriz nula. O termo "indeterminada" é empregado para designar matrizes cujo determinante é nulo, neste caso, tais matrizes não possuem inversa.

A noção de matriz evoluiu e adquiriu uma nova identidade com a memória de Cayley. As matrizes deixaram de ser consideradas apenas como geradora de sistemas de determinantes menores e passaram a ser associadas às leis de um cálculo simbólico e a um teorema notável. A sua identidade também é associada à prática de fatoração de polinômios de matrizes que se baseia na concepção da matriz, ao mesmo tempo, como uma quantidade múltipla (sistema de números) e como uma quantidade simples (BRECHENMACHER, 2006a).

Sylvester, trinta anos depois da introdução do termo matriz, passou a conceber as matrizes em referência a Cayley, em seus trabalhos sobre potências e raízes de substituições lineares e quatérnions (BRECHENMACHER, 2006a).

As matrizes já eram conhecidas por Cayley através dos trabalhos de Sylvester e Cayley já havia definido algumas operações com matrizes em memórias anteriores. No entanto, na memória de 1858, elas são o seu objeto de investigação. A partir das matrizes, questões foram colocadas acerca da possibilidade de formar funções com matrizes 
(potências, polinômios, raiz quadrada, etc.). Além disso, uma prática de fatoração de polinômios com matrizes foi elaborada. Dessa forma, as matrizes funcionaram como o elemento gerador de questões, elas são, portanto, o objeto epistêmico deste episódio e possuem um status bem diferente do episódio anterior. Temos aqui mais um exemplo de uma noção que surge no papel de técnica e se torna o objeto epistêmico de outro episódio.

As questões que poderiam ser colocadas sobre as matrizes dependem do método de gerar respostas, isto é, das técnicas epistêmicas, as ferramentas matemáticas pelas quais Cayley investigou o seu objeto. São elas: o cálculo simbólico aplicado a "quantidades simples", os determinantes, equações polinomiais e a fatoração de equações polinomiais que foi estendida para matrizes.

A configuração epistêmica na qual Cayley trabalhou inclui todo o quadro técnico das transformações lineares, dentro do qual a matriz passou a representar uma "notação abreviada" e as operações com matrizes foram definidas. A herança deixada pela escola algébrica inglesa também está incluída na configuração epistêmica deste episódio.

\section{Conclusões}

Os episódios analisados marcam etapas importantes na história das matrizes. O primeiro (1850/1851) está associado à origem do termo matriz e o segundo (1858), compreende a primeira memória dedicada ao estudo das matrizes, na qual as operações com matrizes são definidas. Comparando os dois episódios, em ambos o objeto epistêmico era conhecido em alguma extensão. No primeiro, a classificação dos pontos de contato entre duas cônicas em quatro tipos já era conhecida e a identificação do tipo de contato já havia sido tratada anteriormente por métodos analíticos. No segundo, as matrizes já eram conhecidas por Cayley a partir dos trabalhos de Sylvester. Além disso, os dois episódios possuem uma técnica epistêmica em comum: os determinantes.

A diferença marcante está no papel das matrizes. Essa noção surgiu em meio às técnicas epistêmicas no primeiro episódio e contribuiu para resolver o problema dos contatos uma vez que a técnica de extração de menores foi baseada em uma representação em forma de tabela, ainda que Sylvester não a tenha usado diretamente. Assim, as matrizes desempenharam o papel de uma técnica epistêmica.

No segundo episódio, a noção de matriz se tornou o objeto epistêmico. A memória de 1858 foi intencionalmente dedicada a estudar este objeto. As matrizes passaram a ser caracterizadas pelas leis de um cálculo simbólico, a um teorema notável e à prática de fatoração de polinômios de matrizes (BRECHENMACHER, 2006a). Elas ofereceram uma nova linguagem, segundo a qual problemas já conhecidos puderam ser tratados de outra forma e novos problemas puderam ser colocados.

Retomando a pergunta colocada na introdução, "O que levou dois matemáticos que viveram na mesma época a conceber a noção de matriz associada a objetos distintos?", uma resposta pode ser formulada comparando-se as configurações epistêmicas. As diferenças começam nos problemas em torno dos quais as pesquisas de Sylvester e de Cayley se desenvolveram, o que tem relação direta com os objetos epistêmicos, uma vez que as questões de pesquisa- os problemas - são formulados em função desses objetos. Além disso, os problemas em cada episódio demandaram técnicas distintas em busca de 
soluções. Os objetos epistêmicos são diferentes e, apesar dos determinantes constituírem uma técnica epistêmica comum, as demais técnicas também são diferentes. A configuração epistêmica dentro da qual a pesquisa está inserida influencia o seu desenvolvimento bem como a forma com a qual os matemáticos definem os seus objetos de estudo.

Observamos que tanto a abordagem de Brechenmacher quanto a de Epple, com a metodologia dos objetos e técnicas epistêmicas, se baseiam na concepção da matemática como uma prática. Ambas preocupam-se em destacar semelhanças e diferenças a partir da comparação de práticas ou episódios de pesquisa. Além disso, ambas as abordagens possibilitam situar no tempo e no espaço as noções matemáticas envolvidas.

Brechenmacher procura situar a noção de matriz dentro do contexto cultural que é próprio de cada episódio, relacionando-a com a prática desenvolvida. Já a perspectiva de Epple possibilita destacar a dinâmica na produção do conhecimento, ao observar as mudanças nos objetos e técnicas ao longo de um ou mais episódios de pesquisa. Vimos que a noção de matriz surgiu como uma técnica epistêmica no primeiro episódio e passou ao papel de objeto epistêmico no segundo. Os determinantes menores surgiram como um objeto epistêmico no primeiro episódio e trocaram de papel no mesmo episódio, ao se tornarem uma técnica que contribuiu para gerar respostas sobre o problema dos contatos. Tais mudanças ilustram como os objetos surgem de forma dinâmica dentro de contextos de pesquisa e como eles se modificam.

Finalizamos, ressaltando a importância da visão compartilhada pelas abordagens historiográficas acima para o ensino de matemática. Sendo a matemática vista como uma atividade dinâmica, seus objetos são construídos dentro de práticas específicas, situadas no tempo e no espaço. Isso quer dizer que os objetos matemáticos têm um início e podem se alterar ao longo do tempo. Essas ideias constrastam com a visão que é promovida pelo ensino dessa disciplina, que apresenta os objetos matemáticos como entidades imutáveis e atemporais. A releitura dos episódios aqui apresentados é parte de uma pesquisa de doutorado (BERNARDES \& ROQUE, 2014), em finalização, cuja proposta de trabalho articula a história da noção de matriz ao ensino de Álgebra Linear.

\section{Bibliografia}

BERNARDES, A. \& ROQUE, T. 2015. Reflecting on meta-discursive rules through episodes from the history of matrices. In: BARBIN, E.; JANKVIST, U. T.; KJELDSEN, T. H. (eds.) History and Epistemology in Mathematics Education Proceedings of the Seventh European Summer University ESU 7.

BRECHENMACHER, F. 2006a. Les matrices: formes de representation et pratiques opératoires (1850-1930). Site expert des Ecoles Normales Supérieures et du Ministère de l'Education Nationale. Paris, 2006. Disponível em:

〈http://www.math.ens.fr/culturemath/index.html>. Acesso em: 11 jul. 2012.

BRECHENMACHER, F. 2006b. Histoire du théorèm de Jordan de la décomposition matricielle (1870-1930). Thèse de doctorat, Ecole des Hautes Etudes en Sciences sociales, Paris. 
CAYLEY, A. 1855. Remarque sur le notation des fonctions algébriques. In: FORSYTH, A. R. The Collected Mathematical Papers of Arthur Cayley, v. 2. Cambridge: University Press, 1889. 185-188.

CAYLEY, A. 1858. A memoir on the theory of matrices. Philosophical Transactions of the Royal Society of London. 148, 17-37.

CAYLEY, A. 1889. James Joseph Sylvester. In: FORSYTH, A. R. 1897. The Collected Mathematical Papers of Arthur Cayley, v. 13. Cambridge: University Press. 43-48.

EPPLE, M. 2004. Knot invariants in Vienna and Princeton during the 1920s: Epistemic Configurations on Mathematical Research. Science in Context. 17, 131-164.

EPPLE, M. 1999. Die Entstehung der Knotentheorie: Kontexte und Konstruktionen einer modernen mathematischen Theorie. Wiesbaden: Vieweq. Apud EPPLE, M. 2004. Knot invariants in Vienna and Princeton during the 1920s: Epistemic configurations on mathematical research. Science in Context. 17, 131-164.

FAUVEL, J., GRAY, J. (eds.) 1987. The History of Mathematics: A Reader. London: MacMillan Press.

FORSYTH, A. R. 1895. Arthur Cayley. The Collected Mathematical Papers of Arthur Cayley, v. 8. Cambridge: University Press.

JANKVIST, U. T. 2009. Using History as a 'Goal' in Mathematics Education. PhD thesis, IMFUFA, Roskilde University, Roskilde.

KJELDSEN, T. H., PEDERSEN, S. A., and SONNE-HANSEN, L. M. 2004. Introduction. In: New Trends in the History and Philosophy of Mathematics. Denmark, University Press of Southern Denmark. 11-27.

KJELDSEN, T. H. 2009. Egg-forms and measure-bodies: Different mathematical practices in the early history of the modern theory of convexity. Science in Context. 22(1), 85-113.

KJELDSEN, T. H. and BlOMHØJ, M. 2012. Beyond motivation - history as a method for the learning of meta-discursive rules in mathematics. Educational Studies in Mathematics. 80. 327-349.

PARSHALL, H. H. 1998. To belong: The role of community in the life and work of J. J. Sylvester. Mathematical Intelligencer. 20. 35-39.

RHEINBERGER, H.-J. 1997. Toward a History of Epistemic Things: Synthesizing Proteins in the Test Tube. Stanford: Stanford University Press. Apud. EPPLE, M. 2004. Knot invariants in Vienna and Princeton during the 1920s: Epistemic configurations on mathematical research. Science in Context. 17, 131-164.

ROQUE, T. 2012. História da Matemática: Uma visão crítica, desfazendo mitos e lendas. Rio de Janeiro: Zahar.

SYLVESTER, J. J. 1850a. On the intersections, contacts, and other correlations of two conics expressed by indeterminate coordinates. In: BAKER, H. F. 1904. The Collected Mathematical Papers of James Joseph Sylvester, v. 1. Cambridge: University press. 119137.

SYLVESTER, J. J. 1850b. Additions to the articles "on a new class of theorems", and "on pascal's theorems". In: BAKER, H. F. 1904. The Collected Mathematical Papers of James Joseph Sylvester, v. 1. Cambridge, University Press. 145-151. 
SYLVESTER, J. J. 1851a. On the intersection of two conics. In: BAKER, H. F. 1904. The Collected Mathematical Papers of James Joseph Sylvester, v. 1. Cambridge: University Press. p. 162-164.

SYLVESTER, J. J. 1851b. An enumeration of the contacts of lines and surfaces of the second order. In: BAKER, H. F. 1904. The Collected Mathematical Papers of James Joseph Sylvester, v. 1. Cambridge: University Press. 219-240.

SYLVESTER, J.J. 1851c. On the Relation between the Minor Determinants of Linearly Equivalent Quadratic Functions. In: BAKER, H. F. 1904. The Collected Mathematical Papers of James Joseph Sylvester, v. 1. Cambridge: University Press. 241-250.

WITMER, R. 1948. Ars Magna, or, The Rules of Algebra. Cambridge: MIT Press.

\section{Aline Bernardes}

Universidade Federal do Estado do Rio de Janeiro UNIRIO e Universidade Federal do Rio de Janeiro - UFRJ - Brasil.

E-mail: $\underline{\text { aline.bernardes@ } @ \text { uniriotec.br }}$

\section{Tatiana Roque}

Universidade Federal do Rio de Janeiro - UFRJ Brasil.

E-mail: tati@im.ufrj.br 